\title{
Spatial Embedding And the Structure of Complex Networks
}

\author{
S. Bullock ${ }^{1}$ and L. Barnett ${ }^{2}$ and E. A. Di Paolo ${ }^{3}$ \\ ${ }^{1}$ School of Electronics and Computer Science \\ University of Southampton, UK \\ ${ }^{2}$ Centre for Computational Neuroscience and Robotics \\ University of Sussex, UK \\ ${ }^{3}$ Department of Logic and Philosophy of Science \\ University of the Basque Country, Spain.
}

March 5, 2010

\begin{abstract}
We review and discuss the structural consequences of embedding a random network within a metric space such that nodes distributed in this space tend to be connected to those nearby. We find that where the spatial distribution of nodes is maximally symmetrical some of the structural properties of the resulting networks are similar to those of random non-spatial networks. However, where the distribution of nodes is inhomogeneous in some way, this ceases to be the case, with consequences for the distribution of neighbourhood sizes within the network, the correlation between the number of neighbours of connected nodes, and the way in which the largest connected component of the network grows as the density of edges is increased. We present an overview of these findings in an attempt to convey the ramifications of spatial embedding to those studying real-world complex systems.
\end{abstract}

\section{Introduction}

The new science of networks [21,4] aims to generate insights into complex systems by representing them as graphs (networks) comprising a number of nodes (parts, elements, components, individuals) connected by edges (connections, interactions). Once represented in this way, graph-theoretic metrics and analyses can be used to characterise the structural organisation of the target complex systems. These characterisations are helpful in that they can suggest mechanisms of growth [1], processes of reconfiguration [27], or other explanatory accounts of complex system behaviour.

The insights, methods and measures derived from complex networks science may be applied to a vast range of potential target systems: telecommunication networks, social affiliation networks, transport networks, epidemics, neural networks, metabolic pathways and genetic regulatory networks, among many others, see, e.g., [14]. It is attractive to think that the implications of abstract models of complex networks might generalise across different domains. This is one of the reasons behind the excitement generated by complex networks. However, it may be expected that real progress on specific applications might not just require the addition of domain specific knowledge to a general insight, but also sometimes will force researchers to question whether there are important aspects of complex networks that need further investigation in order to make them more widely applicable. Many of the real systems mentioned present a spatial structure and often this structure constrains the possible connectivity and interactions between nodes in the network and their activity. This motivates an investigation into the role of space on complex networks. 
Traditionally, one popular approach has been to define classes of random graphs, so called because they are generated by a random process. By characterising the properties of a set of random graphs and explaining how these properties vary with parameters such as connection density, number of nodes, amount of random rewiring of connections, etc., networks science can reveal what the generic properties of such a graph can be expected to be.

For instance, Erdős and Rényi [9, 10, 5] defined a very simple class of random network in which each pair of nodes is connected with probability $p$. Amongst other things, they showed that as the density of network connections is increased (i.e., as $p$ grows), their networks reach a connection density at which they pass through a very rapid step change in their overall organisation. One such "phase transition" involves the onset of a giant component: a sub-set of nodes that are either directly or indirectly connected to one another by network paths and between them comprise the vast majority of the network. Below a threshold value of $p=1 / n$ (where $n$ is the number of network nodes) such a giant component is almost certainly absent from an Erdós-Rényi network, which will tend to comprise many disconnected fragments. Above this threshold, a single giant component is almost certainly present.

Similarly, Watts and Strogatz [27] show that gradually randomly re-wiring a regular lattice can quickly generate a "small world" graph that retains the high clustering that is characteristic of the original lattice (the neighbours of a node are likely to themselves be neighbours ${ }^{1}$ ) but also enjoys the relatively short path lengths separating arbitrary pairs of nodes that is characteristic of Erdős-Rényi random networks.

The third major random graph model is due to Barabási and Albert, who proposed a random preferential attachment process capable of growing a network that exhibits a scale-free degree distribution, meaning that the expected number of neighbours of a randomly chosen node (its expected degree) follows a power law distribution [1]. Unlike both the Erdôs-Rényi and WattsStrogatz models, where a node's degree can be expected to be close to the network's mean degree, Barabási-Albert's process ensures that many nodes have very low degree (they are peripheral nodes connected only to a very small number of neighbours) while a few have very large degree (hubs directly connected to a large proportion of the network).

There are of course many variants of these models, and alternative random graph models $[5,21,4]$. However, in the context of the burgeoning networks science literature, the role of spatial embedding has, arguably, been somewhat neglected given that the vast majority of realworld complex systems are subject to the constraints that result from being spatially extended (including the problem system identified with the birth of graph theory itself: Euler's Seven Bridges of Königsberg). Both the Erdős-Rényi and Barabási-Albert models consider nodes to be a-spatial, with no relationship between nodes other than whether they are connected or not. The Watts-Strogatz model is different in that it commences with a lattice that can be thought of as a set of points arranged regularly in some space and connected to their nearest neighbours, and then proceeds to erode this spatial organisation. However, little attention is paid to the spatiality of small worlds, per se.

This is despite the fact that the small-world notion originated in a set of social network experiments that were explicitly geographical in spirit. Milgram [26] invited participants in his seminal experiment to send a package to a recipient identified by name, address and occupation, but only via a chain of people known on first-name basis. Results of the experiment showed that when a package arrived at its ultimate destination it had passed through on average between five or six intervening people who had been able to combine their social and geographic knowledge to achieve a remarkably short path length between initial sender and final recipient (but see [13] for a critique of Milgram's study).

Subsequent studies of spatially embedded networks have tended to be domain specific and

\footnotetext{
${ }^{1}$ In this paper, there is scope for confusion in the meaning of terms such as "neighbour" or "near" or "close", each of which might be interpreted either with respect to the spatial distance between nodes or with respect to the connectivity of the network. Here we will refer to a node's neighbours as those nodes that are directly connected to it on the network. When necessary this will be explicitly distinguished from nodes that share the same spatial locale. Similarly, a "path" between two nodes will be always be interpreted in terms of traversing connections on the network, rather than moving directly through space.
} 

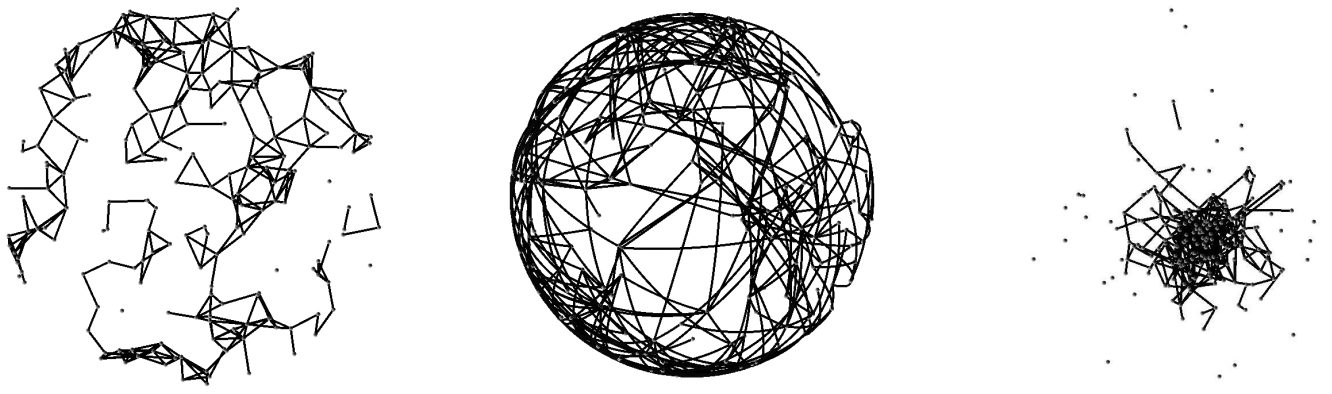

Figure 1: Examples of spatially embedded random networks, constructed a) uniformly on a disc, b) uniformly on a sphere, c) non-uniformly on a plane.

aimed at modelling some spatial aspect of network formation [28, 3, 19, 29, 17, 16, 18, 25, 15] or addressing somewhat restrictive spatial embeddings [22, 8, 12, 24]. The current paper aims, therefore, to take a more general and fundamental perspective on the constraints on network structure implied by spatial embedding, drawing heavily on a recent technical exposition [2]. ${ }^{2}$

\section{Overview}

By a spatially embedded network in the broadest sense we shall mean the following: network nodes reside in a metric space and the likelihood of a pair of nodes being connected depends in some way on the spatial distance between them. The space could be a "real" (Euclidean) space or perhaps some more abstract space suggested by the problem or model domain (e.g., a political spectrum ranging over far-right, right-wing, centrist, left-wing and far-left ideologies). We generally imagine that network nodes which are nearby in space have a better chance of being connected than distant nodes; that is, connection probability decays with distance.

Spatially embedded networks in the above sense have traditionally been studied in the (restricted) form of Random Geometric Graphs (RGGs) [8, 22]. In these networks, nodes are distributed uniformly at random over some Euclidean space and pairs are connected only if they fall within a characteristic distance of each other. Studies of RGGs have generated some insight into their structure, including the relationship between the amount of clustering and the dimensionality of the space, and the manner in which a giant connected component appears as the connection density is gradually increased. The interested reader can find an overview of these results in [12]. In [2], Barnett et al. introduced a generalisation of RGGs, the Spatially Embedded Random Networks (SERN) model, where the embedding space is no longer necessarily Euclidean, connection probability decay is not restricted to a simple distance cutoff ${ }^{3}$ and, crucially, the distribution of nodes is no longer required to be uniform in space. It is the latter feature-the possibility of spatial inhomogeneity and the consequent variability in the likelihood of different pairs of nodes being connected together - which turns out to have a decisive impact on the structure of the resulting networks, e.g., fig 1. In analysing the properties of the SERN model (and in particular the behaviour of various "motif moments", see fig 2), we were able to demonstrate that:

- Where the spatial distribution of nodes is homogeneous (i.e., there is maximal spatial symmetry) the degree distribution of a spatially embedded random network is equivalent to that

\footnotetext{
${ }^{2}$ Unless explicitly indicated, evidence and/or arguments supporting the claims and results reported in this paper can be found in [2].

${ }^{3}$ While any pair of SERN nodes are either connected, or not, in general these connections are determined probabilistically, not deterministically, and we may be required to consider expectations of graph properties calculated over an ensemble of networks generated using a particular distance decay function for a particular spatial distribution of nodes. RGG graphs are a degenerate case of this scheme in which the probability of connectance is unity for nodes separated by a distance less than some value, $d$, and is zero otherwise.
} 

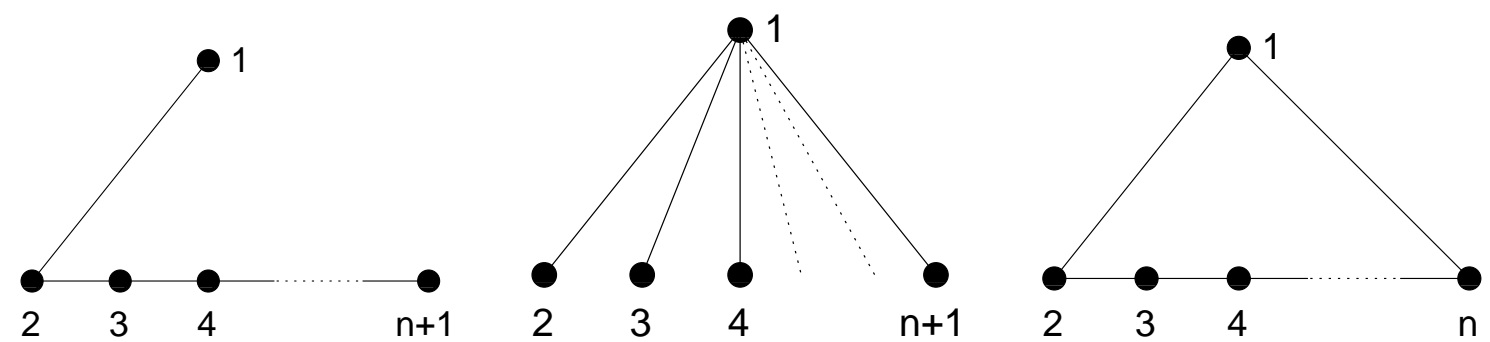

Figure 2: Examples of, respectively, chain, fan and loop motif "moments" implicated in the analysis of spatially embedded random networks. A motif is a distinctive, recurrent structural network element. A moment is an average of a product of random variables. Motif moments, averaged over an ensemble of networks, are useful and intuitive building blocks in terms of which various network statistical features may be expressed. For example, the 'chain', 'fan' and 'loop' motifs illustrated appear in the expression for degree correlation.

of a non-spatial random graph.

- Similarly, as the number of connections in such a homogeneous spatial graph is increased a phase transition to a giant component may be observed, as in non-spatial random graphs.

- However, if there is spatial inhomogeneity in the distribution of nodes then there is evidence to suggest that no phase transition to a giant connected component occurs; rather, we conjecture that the expected size of the largest component will grow smoothly as more edges are added to the network.

- Spatially embedded random networks tend to exhibit assortative degree correlation, with highly connected nodes tending to be more likely to be connected to each other, and this correlation is boosted by inhomogeneity in the spatial distribution of nodes.

- Spatially embedded random networks may exhibit a scale-free degree distribution, but this requires that there exist a singularity in the spatial distribution of nodes, i.e., a point in space near which the density of nodes increases without limit.

- Under reasonable assumptions, we conjecture that random geometric graphs (and a broader class of lattice-like graphs to which they belong) cannot be small worlds. However, more generally, spatially embedded small worlds can exist, and indeed spatial rewiring schemes can create small world properties in arbitrary clustered networks.

In the next section we expand on these results, before discussing some caveats and qualifications that have to be borne in mind. In this paper our intention is to explicate the way in which spatial embedding confers certain properties on graphs without resorting to mathematical proofs (which can be found in the original paper [2]).

\section{Structural Properties of Spatially Embedded Random Net- works}

\subsection{Homogeneity vs. Inhomogeneity}

Upon setting out to explore the general properties of spatially embedded networks, an obvious place to start is a network constructed over a random spatial distribution of otherwise featureless nodes. For instance, we might scatter nodes across a square patch of space and connect together those that are closer together than some threshold distance, not caring about any other property of the nodes. Of course, points near the boundary of the square patch might be non-standard 
in their properties, so we might restrict our attention to part of the space that is far from the boundary. Or perhaps, rather than consider a square patch, we might consider points randomly distributed over a sphere which has no problematic boundaries ${ }^{4}$. We might also avoid considering spaces that have holes in them or other incongruities, and we might also be sure to distribute the nodes according to a uniform random distribution rather than a distribution that tends to put more points in some areas than in others. By considering many networks constructed over many random distributions of nodes we can reach expectations of generic network properties, rather than be distracted by particular peculiarities of specific instances of an individual random distribution and its associated network(s).

The epitome of this approach is the consideration of what we shall call homogeneous spatial networks: networks constructed over a distribution of nodes that is maximally spatially symmetrical. What does this mean? Consider a set of points distributed uniformly over a sphere. From whichever angle we choose to look at it, this distribution of points tends to look the same. If we were to stand on the sphere at the location of one point and be transported to the location of another point chosen at random, we would tend not to notice the difference.

When we consider such networks, in some respects they resemble Erdôs-Rényi random graphs (E-R graphs) constructed over nodes that have no spatial location-in an E-R graph the chance of any two nodes being connected is equal. For instance, if we count what proportion of network nodes have no neighbours, or only one neighbour, or two neighbours, or three or have 10 neighbours (i.e., we calculate the network's degree distribution), we find that, whether we are describing a nonspatial E-R graph or a homogeneous spatial network, the degree values follow the same Poisson distribution ${ }^{5}$.

This makes sense since the Poisson distribution is known to represent the frequency with which a number of independent events occur in some unit of time, or, in this instance, within some unit of spatial area. "Will a node have three neighbours or two neighbours, or some other number?", turns out to be the same kind of question as "Will this week's lottery have three winners or two, or some other number?".

However, despite their congruent degree distributions, homogeneous spatial networks are not equivalent to Erdôs-Rényi graphs. In an E-R graph the chances that two of a node's neighbours are connected is the same as the chance that one of its neighbours is connected to any other randomly chosen node (since any pair are connected with probability $p$ ). However, in a homogeneous spatial network, since nodes are connected to those that are nearby, a node's neighbours tend to be close together and are therefore more likely to be connected to each other resulting in more clustering than in an equivalent Erdôs-Rényi graph.

The properties of a particular class of homogeneous spatial networks and whether they are equivalent or not to properties of an E-R graph have been explored in work on Random Geometric Graphs [8, 22]. Here we are more interested in exploring properties of the more general class of inhomogeneous spatial networks. This is motivated primarily by the fact that the vast majority of extant real-world networks fall into this category having variously, edges, holes, moreor-less sparse/dense regions, non-uniform distributions of nodes, or other factors that introduce heterogeneity.

Moreover, inhomogeneous spatial networks offer a second important advantage in a more fundamental sense. For homogeneous spatial networks, as was described above, there is no real difference between one place and another - every location in the space is equivalent. But, a critical property of spatial systems must surely be that there are consequences associated with where you are located. Even when a number of nodes are scattered at random on a sphere, and there is no tendency to preferentially scatter them in some places more than in others, the scattering will tend to result in more or less sparsely populated regions "accidentally". Of course, on average and in the limit, all such nodes will tend to find themselves in identical circumstances. However,

\footnotetext{
${ }^{4}$ In fact it turns out that the existence of a boundary is structurally significant only in restricted circumstances [2].

${ }^{5}$ In fact (in the context of a particular class of spatial network) it can be demonstrated that a Poisson degree distribution may only arise when nodes are distributed uniformly in space. This highlights the impact of spatial inhomogeneity on network structure.
} 
in every single instance of such a system, some nodes will tend to find themselves in a relatively dense patch of neighbours, while others will find themselves more spatially remote from their nearest neighbours. This inhomogeneity in the distribution of nodes is thus fundamental to spatially distributed systems, and it will tend to be reflected in the network of connections between nodes if spatial distance between them influences the likelihood of a connection being formed and maintained.

\subsection{Giant Components}

One respect in which introducing inhomogeneity in the spatial distribution of nodes has a significant impact is the onset of a giant component in the network as the density of network edges is increased.

If we start by considering a population of nodes with no edges between them, an empty network, and gradually increase the number of edges that the network contains, tending to connect together nodes that are closer together, we initially see a growing number of connected pairs of nodes. After a while we begin to see network "fragments" each comprising a small number of sparsely connected nodes. Over time these fragments tend to grow in size and eventually coalesce as edges are added that link together previously disconnected fragments. If the initial distribution of nodes in space is homogeneous, then there comes a critical value of edge density when the network transitions from comprising a large number of isolated fragments to comprising a single giant connected component that contains the vast majority of nodes: i.e., there is a "phase transition" in the onset of a giant component. This result was originally shown in the context of E-R graphs, and has been shown to generalise to homogeneous spatial networks in simulations [8].

However, our simulations support a conjecture that there will be no such phase transition to the extent that there is initially any inhomogeneity in the spatial distribution of nodes. Rather, as edge density increases, the size of the largest connected component of the network grows more smoothly, and the giant component does not arise abruptly, but arrives more gradually. This can be understood by noting that the phase transition relies on coalescence to happen at the same rate across the entire population.

In order for the size of the largest component to remain initially small while the first edges are added but then to rapidly transition to a giant component of much larger size, network fragments must first grow in size without becoming connected to each other, reaching a point at which a few additional edges inter-connect a large number of such fragments. In order to reach such a point no network parts can be easier to connect together than others as this would smooth out the growth of the largest component and prevent the phase transition.

Consider a multiple-choice test being taken by a class of identical student "clones" that are identically capable and identically well-prepared and identically well-rested, etc. We might expect them all to complete the exam at roughly the same time. As we watched the class at work, we would see an abrupt transition in the number of students that had completed the test-before the rapid transition most would be working on the test, and just afterwards the vast majority would have stopped work. However, in a real classroom students vary in many ways and we see a much smoother rate of completion-not a phase transition. A spatially inhomogeneous network exhibits just this kind of variability in the propensity to become well-connected with some parts benefitting from the effects of the inhomogeneity and some parts suffering from it.

The implications are significant. E-R graphs and homogeneous spatial networks of varying size and edge density are likely to be observed to fall into one of two classes: either they comprise many small fragments, or they feature a single giant component. Since the transition between these two classes of network is very abrupt we are unlikely to see networks that fall in between the two classes. However, where real-world networks are spatially embedded and inhomogeneous, we should expect to encounter networks that do fall between the two classes: networks that feature a number of disconnected components that vary in size, the largest of which may variously be small, moderately sized, or giant. 


\subsection{Degree Correlation}

We have already mentioned that spatial graphs can expect to exhibit a higher degree of clustering than equivalent non-spatial graphs, i.e., a node's neighbours have an increased chance of being connected together in a spatial graph. But can we say more about the character of a node's neighbours? In particular, if a node has many neighbours, will that make it more or less likely that its neighbours are also well-connected?

The correlation between a node's degree (its number of neighbours) and the average degree of those neighbours is called degree correlation or assortativity. In a positively assortative network, high-degree nodes tend to be connected together. In a negatively assortative (or disassortative) network high-degree nodes tend to be connected to low-degree nodes. Both kinds of assortativity may be observed in real-world networks. For instance, collaborative networks such as co-authorship graphs and actor-collaboration graphs may exhibit positive degree correlation, while some technological and biological systems such as the Internet, world-wide-web, protein networks, neural nets, and food webs may exhibit negative degree correlation [20].

By contrast, Erdős-Rényi random graphs, Barabási-Albert preferential attachment graphs and Watts-Strogatz small worlds all have zero degree correlation, there being no consistent relationship between the degree of connected nodes. The same is not true of spatial networks, which tend to exhibit positive (assortative) degree correlation even when they are homogeneous. In the simplest homogeneous case, the magnitude of this correlation is equal to the degree of clustering in the network. Moreover, introducing inhomogeneity into such spatial networks boosts this correlation, with increasing inhomogeneity leading to increasingly positive assortativity.

It is easy to see why spatial graphs would exhibit assortative degree correlation. In order that a node achieve higher than average degree, it must tend to be the case that it is closer to its neighbours than is the average node-it is in a well-populated patch. Since the nodes to which it is connected will tend to be close by, they will tend to also benefit from the local population density and will themselves tend to have a higher than average degree as a consequence. Conversely, a low-degree node will tend to be found in a low-density patch, connected to a small number of similarly disadvantaged nodes. As a result, a node's degree will tend to be a good estimate for that of its neighbours. Of course, to the extent that a spatial network is inhomogeneous, there will be more disparity between its dense and sparse patches, exacerbating this assortativity effect.

It would be interesting to relate this theoretical result to the empirical findings mentioned above. To what extent might the impact of spatial constraints on network formation and maintenance account for the observed positive and negative degree correlations in natural, social and engineered systems?

\subsection{Scale-Free and Small-World Spatial Networks}

Can a set of nodes that are identical save for their location in space be connected together solely on the basis of the distances between them in order to arrive at a graph that exhibits scale-free or small-world structure? In both cases the answer is no if we limit ourselves to considering the class of random geometric graphs but yes if we are considering the more general class of spatially embedded random networks.

First, can a scale-free degree distribution arise purely as a consequence of nodes tending to be connected when they are close in space? Recall that a degree distribution is said to be scale-free when the probability that a randomly chosen node has a particular degree follows a power law. In such cases, many nodes have very low degree, but a small minority have extremely high degree. It can be shown that a random geometric graph (where nodes are distributed uniformly at random in space) cannot exhibit this type of structure-where nodes tend to be equi-distant from their neighbours, there tend to be no large disparities in connectivity.

However, scale-free spatial networks are possible where node distribution is inhomogeneous. In particular, consider nodes that are arranged such that node density increases at an accelerating rate as we approach a particular point in space, and the density at that point is in effect infinite. If we generate a population of nodes according to this distribution and then connect together pairs 
of nodes that are close together in space, then a scale-free network can result.

This is an example of "power law in, power law out". The scale-free degree distribution reflects our deliberate choice to distribute the nodes in space in a particular "scale-free" manner. It may of course be possible to generate a scale-free spatial network without demanding a singularity in the distribution of nodes if we allow a more sophisticated method of connecting nodes, perhaps one that makes some use of the "rich get richer" dynamic that is brought about by "preferential attachment" [1].

What of small-world networks, in which, simultaneously, there is significant clustering (like a lattice, or spatial network) but also the path between any two randomly chosen nodes tends to involve only a small number of intervening edges (like a totally random graph)? First, we can show that, as for scale-free networks, small worlds cannot occur in random geometric graphs. Recall that for this class of graph, nodes are only connected if they are separated by a distance less than some threshold. As such, "long-range" connections are outlawed. While clustering is significant in such graphs, the average path length separating network nodes tends to be long-some pairs of nodes are separated by a significant spatial distance and consequently getting from one to the other requires a large number of network "hops".

We can understand that this is prohibitive of small-world structure by noting that the classical way to construct a small world is to start with something like a random geometric graph (a lattice) and alter it (by randomly rewiring a few edges) such that it contains new edges that would be illegal under the original RGG wiring scheme- they tend to break connections between pairs of near-by nodes and introduce connections between arbitrary pairs of nodes that, at least for the first few rewirings, tend to be separated by a much longer distance.

However, it is possible to construct spatial small worlds for the less restrictive class of spatially embedded random networks. Specifically, we must consider graphs where, rather than using a distance threshold to truncate connectivity, we allow connectivity to decay with distance probabilistically. For example, we could construct a scheme where there is a base probability $q$ that any pair of nodes are wired together, but also an additional probability $1-q$ that nodes are wired together if they are separated by a distance less than some threshold $d$. For this scheme, as we consider larger and larger networks, we see significant clustering and low mean path length-i.e., small worlds. It is possible to interpret this scheme as a spatial analogue of the original WattsStrogatz model with lattice-like local connectivity ensured by the $1-q$ term and longer-range connections introduced by the $q$ term.

Interestingly, it can be shown that this approach to generating a small world can be used for any lattice-like network (i.e., a network with strong clustering but long path lengths) and that it brings about small world properties while preserving many structural aspects of the original network, such as scale-free or Poisson degree distribution, for instance. By introducing just enough arbitrary connections to any spatial network, short path length can be achieved without compromising clustering, and without radically altering the network's connectivity structure. In some sense, this emphasizes the essentially spatial nature of the original Watts-Strogatz model.

\section{Caveats and Qualifications}

The results described above are true for various idealised mathematical representations of realworld systems. To what extent should we understand these results as transferring to the real world? Unfortunately, several caveats and qualifications must be borne in mind.

First, unlike the mathematical objects explored here, the structure of any real-world spatial network is not wholly determined by the spatial organisation of its nodes. The presence and absence of relationships amongst the parts of a real-world system can be influenced by more than the distance between these parts. Shared affinities, shared histories, and other contingencies will also play a part. In the models explored here we are interested in understanding what might be expected to come about purely as a consequence of spatial proximity between nodes influencing their connectivity. Whatever structures tend to arise in these models can be understood as a kind of baseline organisation that we can expect to arise "for free" in spatially embedded networks [6]. 
Where real-world spatial networks reveal the kind of structures exhibited in these models, it is in some sense unremarkable. Conversely, where such networks depart from this type of organisation it may be at significant cost, or as a result of significant (and possibly interesting) organising processes that are not captured in the models presented here.

Second, in order to reach the results reported in the previous section, it has been necessary to idealise spatial networks in various ways. For mathematical tractability, we have followed others in sometimes considering a space to be populated uniformly or by an infinite number of nodes. It is clear that real-world networks are not like this. There are two main issues here, one more technical and one more practical. First, we have made different kinds of idealisation at different points in order to achieve particular insights. For instance, in this paper, we have only tended to distinguish between "homogeneous" and "inhomogeneous" spatial networks, which has tended to disguise the more subtle distinctions made between different ways of idealising homogeneity in terms of, e.g., uniformity, continuity, and spatial homogeneity, and the necessity to restrict analyses to special cases such as generalisations of random geometric graphs. In order to fully understand the scope of each result, a firm grasp of these idealisations is necessary. Perhaps more significantly, any and all of these idealisations will mean that, to some extent, the relatively clean statements that we are able to make about the ideal mathematical objects will not carry over to real-world networks. Unfortunately, it is inherently difficult to know how these idealisations play out in terms of the transferability (or not) of mathematical results to the real world. ${ }^{6}$ For a full account of the idealisations that we have made please see [2].

A third set of more more generic concerns are also associated with the networks science approach to complex systems exemplified here. First, real-world networks are not static objects in the way that we (and many other networks scientists) have treated them here. Real networks arise as a consequence of processes that take place in the real world. Typically these processes do not stop once a certain number of edges or nodes are in place. Rather, real-world networks are constantly being brought about by ongoing processes. They are thus inherently dynamic and any consistent structural properties that they exhibit are dynamically maintained in the face of perturbation and erosion, an aspect which has not been treated in this paper (or in many of the papers that we cite, e.g., [5, 27, 1]). Furthermore, the empirical basis for comparing results from networks science with those from experimental studies of real-world networks is somewhat suspect since we can only sample real-world networks, and report the properties of the samples. If our sampling method is biased in some way that our samples will not be accurate reflections of the underlying real-world network. They will be distorted. At times it has not been recognised that a fair comparison between the properties of a model network and evidence from real world networks must address this issue of sampling, see, e.g., [11, 7] for a treatment of this issue in the context of social networks. Finally, it must be remembered that "networks" do not necessarily exist in the real world. Cables exist in the real world, connecting power stations, sub-stations, etc., but the "power network" is not accounted for by a network of nodes and the edges between then, since the relevant technological infrastructure is structurally coupled with a wider context of inputs, outputs, policies, drivers, and adjacent "networks". To equate the power network with a graph representing the lines of power distribution is to idealise and simplify the real world. Similarly, children in a playground exist, but the social network representing their relationships is a conceptual framework, a theoretical postulate, or, more plainly, just an idea. Of course the promise of networks science is that there will be many cases in which a particular network idea will play a key role in how we make sense of the phenomena associated with it, to the extent that we start assigning it some kind of causal efficacy. In such a case the network idea is no different from other ideas that only seem more concrete because of their identification with material structures (e.g., cables and power stations).

\footnotetext{
${ }^{6}$ The same problem is true for computational approaches. While particular idealisations might be side-stepped by using numerical methods, or simulations, the issue of idealisation itself cannot be avoided.
} 


\section{Conclusions and Future Work}

We have aimed to elucidate the contribution that spatial embedding makes to the structure of networks, taking as our starting point a tradition of analysing the properties of random graph ensembles. By considering a more general model of spatial networks that allows for different relationships between proximity and connectivity and for inhomogeneous distributions of nodes in space, we have been able to show some of the ways in which spatial networks can be expected to differ from their non-spatial cousins. One motivation for such analysis is to achieve a new class of null models of graph structure that control for the (extremely pervasive) influence of spatial embedding. Another is to achieve a more profound understanding of the role of spatial constraints in enabling complex organisation.

In achieving these results we have been able to demonstrate circumstantial evidence that our approach (in terms of identifying the "right statistics" to analyse spatial networks, i.e., motif moments, conditional mean degree, etc., [2]) is on the right track. However, there are significant gaps in the analysis that are worth flagging here. First, we have so far considered graphs that are first constructed and then analysed as static objects, rather than addressing the relationship between spatial processes and the dynamic network structure that they bring about. Second, we have so far considered spatial distributions of nodes that do not themselves exhibit significant structure, in terms of, for instance, separate clusters (something that might be remedied by generating spatial distributions of nodes using spatial point processes, [23]). A satisfying treatment of these two issues would perhaps relate them to offer an understanding of how spatial and network structure mutually inform one another over the lifetime of a real-world spatially embedded system.

\section{References}

[1] A.-L. Barabási and R. Albert. Emergence of scaling in random networks. Science, 286:509, 1999.

[2] L. Barnett, E. Di Paolo, and S. Bullock. Spatially embedded random networks. Phys. Rev. E, 76(5):056115, 2007.

[3] M. Barthélemy. Crossover from scale-free to spatial networks. Europhys. Lett., 63:915, 2003.

[4] S. Boccaletti, V. Latora, Y. Moreno, M. Chavez, and D.-U. Hwang. Complex networks: Structure and dynamics. Phys. Rep., 424:175-308, 2006.

[5] B. Bollobás. Random Graphs. Academic, 1985.

[6] S. Bullock and C. L. Buckley. Embracing the "tyranny of distance": Space as an enabling constraint. Technoetic Arts, 7:141-152, 2009. Special issue on Living Buildings: Plectic Systems Architecture.

[7] D. P. Croft, R. James, and J. Krause. Exploring animal social networks. Princeton University Press, 2008.

[8] J. Dall and M. Christensen. Random geometric graphs. Phys. Rev. E, 66:016121, 2002.

[9] P. Erdôs and A. Rényi. On random graphs. I. Publicationes Mathematicae, 6(290-297), 1959.

[10] P. Erdôs and A. Rényi. The evolution of random graphs. Magyar Tud. Akad. Mat. Kutató Int. Közl., 5(17-61), 1960.

[11] D. W. Franks, G. D. Ruxton, and R. James. Sampling animal association networks with the gambit of the group. Behavioral Ecology and Sociobiology, 2009. DOI 10.1007/s00265-0090865-8.

[12] C. Herrmann, M. Barthélemy, and P. Provero. Connectivity distribution of spatial networks. Phys. Rev. E, 68:026128, 2003. 
[13] J. S. Kleinfeld. The small world problem. Society, 39(2):61-66, 2002.

[14] T. G. Lewis. Network Science: Theory and Applications. Wiley, 2009.

[15] Q. Lu, G. Korniss, and B. K. Szymanski. Naming games in spatially-embedded random networks. Proceedings of the 2006 American Association for Artificial Intelligence Fall Symposium Series, Interaction and Emergent Phenomena in Societies of Agents, page 148, 2006.

[16] S. S. Manna, G. Mukherjee, and P. Sen. Scale-free network on a vertical plane. Phys. Rev. E, 69:017102, 2004.

[17] S. S. Manna and P. Sen. Modulated scale-free network in euclidean space. Phys. Rev. E, 66:066114, 2002.

[18] S. Muthukrishnan and G. Pandurangan. The bin-covering technique for thresholding random geometric graph properties. Proceedings of the ACM-SIAM Symposium on Discrete Algorithms (SODA), 2005.

[19] G. Németh and G. Vattay. Giant clusters in random ad hoc networks. Phys. Rev. E, 67:036110, 2003.

[20] M. E. J. Newman. Assortative mixing in networks. Phys. Rev. Lett., 89(208701), 2002.

[21] M. E. J. Newman. The structure and function of complex networks. SIAM Review, 45(2):167256, 2003.

[22] M.D. Penrose. Random Geometric Graphs. Oxford University Press, 2003.

[23] B. D. Ripley. Modelling spatial patterns. J. R. Stat. Soc. Ser. B-Stat. Methodol., 39:172-212, 1977.

[24] A. F. Rozenfeld, R. Cohen, D. ben Avrahamand, and S. Havlin. Scale-free networks on lattices. Phys. Rev. Lett., 89:218701, 2002.

[25] A. Scirè, I. Tuval, and V. M. Eguíluz. Dynamic modeling of the electric transportation network. Europhys. Lett., 71:318-324, 2005.

[26] J. Travers and S. Milgram. An experimental study of the small world problem. Sociometry, $32(4): 425-443,1969$.

[27] D. J. Watts and S. H. Strogatz. Collective dynamics of 'small-world' networks. Nature, 393:440-442, 1998.

[28] B. Waxman. General formalism for inhomogeneous random graphs. IEEE J. Sel. Areas Commun., 6:1617, 1988.

[29] S.-H. Yook, H. Jeong, and A.-L. Barabási. Modeling the internet's large-scale topology. PNAS, 99:13382-13386, 2002. 